\title{
Revisiones Sistemáticas: definición y nociones básicas
}

\section{Systematic Reviews: definition and basic notions.}

\begin{abstract}
Begoña Moreno ${ }^{2,3}$, Maximiliano Muñoz ${ }^{3}$, Javier Cuellar ${ }^{3}$, Stefan Domancic ${ }^{1,2}$, Julio Villanueva ${ }^{1,2,3^{*}}$
1. Departamento de Cirugía y Traumatología Bucal y Maxilofacial, Facultad de Odontología, Universidad de Chile, Santiago, Chile.

2. Unidad de Odontología Basada en Evidencia, Centro Cochrane, Santiago, Chile

3. Unidad de Cirugía Maxilofacial, Hospital Clínico San Borja Arriarán. Santiago, Chile

* Correspondencia autor. Julio Villanueva | E-mail: javm@uchile.cl | Teléfono: +56 229781841 | Dirección:Sergio Livingstone P 943 Independencia (8380492). Santiago de Chile Trabajo recibido el 12/02/2018. Aprobado para su publicación el 11/10/2018

RESUMEN

El desarrollo de la Odontología Basada en Evidencia ha generado la necesidad constante de actualización e información por parte de los profesionales ${ }^{(1)}$. Ante esto, los artículos actualizados que sintetizan información y poseen altos estándares, son las mejores fuentes de evidencia, siendo para ello las revisiones sistemáticas las mejores herramientas ${ }^{(2)}$. Éstas, corresponden a resúmenes claros y estructurados de la información disponible orientada a responder una pregunta clínica específica ${ }^{(3)}$. Poseen un proceso de elaboración estructurado que comienza con el planteamiento de una pregunta clínica específica, con los cuales se realizará la búsqueda en las bases de datos. Una vez obtenida la información se deben seleccionar los artículos y, a partir de los seleccionados, se obtendrán los datos y se realizarán los análisis críticos y estadísticos de la información, finalmente exponiendo los resultados del trabajo.
\end{abstract}

PALABRAS CLAVE:

Revisión sistemática; Odontología Basada en evidencias; Práctica clínica basada en evidencia.

Rev. Clin. Periodoncia Implantol. Rehabil. Oral Vol. 11(3); 184-186, 2018.

\section{ABSTRACT}

The development of Evidence Based Dentistry has generated the constant need for updating and information from professionals ${ }^{(1)}$. Given this, updated articles synthesizing information and with high standards are the best sources of evidence, being systematic reviews the best tools ${ }^{(2)}$. Systematic reviews are clear and structured summaries of the available information aimed at answering a specific clinical question ${ }^{(3)}$. They have a structured elaboration process that begins with the presentation of a specific clinical question, which will be used for the search in the databases. Once the information has been obtained, the articles must be selected and, starting from the selected ones, the data will be obtained and the critical and statistical analysis of the information will be carried out. Finally, the results of the work will be exposed.

KEY WORDS:

Systematic review; Dentistry-based evidence; Evidence-Based Practice.

Rev. Clin. Periodoncia Implantol. Rehabil. Oral Vol. 11(3); 184-186, 2018.

\section{INTRODUCCIÓN}

El mundo actual y su proceso de globalización han generado la aparición creciente y constante de nueva información, plasmada en múltiples artículos y publicaciones. Esta realidad también ha involucrado a las ciencias biomédicas que han observado en los últimos años un aumento de artículos que acreditan el uso de terapias y tratamientos, avalando sus usos. Es por esto que los profesionales de la salud, dentro de los que se incluye a los odontólogos, deben estar informados y permanecer constantemente actualizados a través de las fuentes de información disponibles y sus usos, para lo cual nace la Odontología basada en evidencia (OBE). La OBE es definida por la Asociación Dental Americana como "una aproximación a la salud oral que requiere la integración juiciosa de evaluaciones sistemáticas de evidencia científica clínicamente relevante, en relación al estado e historia médica y odontológica del paciente, considerando la experiencia clínica del dentista y las necesidades y preferencias del paciente"(1).

Dado el gran número de artículos y publicaciones, la manera más sencilla y completa de dar uso a dicha información es mediante una compilación de la información. Para poder responder dicha necesidad, es que se desarrollan las revisiones sistemáticas, cuyo fin último es ser una herramienta práctica en la toma de decisiones clínicas ${ }^{(2)}$.

\section{Qué es una revisión sistemática}

Las revisiones sistemáticas son resúmenes claros y estructurados de la información disponible orientada a responder una pregunta clínica específica(3). Dado que están constituidas por múltiples artículos y fuentes de información, representan el más alto nivel de evidencia dentro de la jerarquía de la evidencia (Figura 1).

Las revisiones sistemáticas se caracterizan por tener y describir el proceso de elaboración transparente y comprensible para recolectar, seleccionar, evaluar críticamente y resumir toda la evidencia disponible con respecto a la efectividad de un tratamiento, diagnóstico, pronóstico, etc. ${ }^{(3)}$

Como se ha mencionado, las revisiones sistemáticas pueden responder preguntas de tratamiento, diagnóstico o pronóstico, donde la diferencia radica principalmente en los estudios primarios que serán incluidos y evaluados. Dentro del universo de preguntas, la mayoría corresponde a preguntas de tratamiento por lo que nos abocaremos principalmente a ellas ${ }^{(4)}$.

\section{Cómo elaborar una revisión sistemática}

El proceso de confección de una revisión sistemática comienza con el planteamiento de una pregunta clínica específica y estructurada que determinará los términos que serán utilizados en la búsqueda en las bases de datos y el tipo de artículos útiles para responder dicha pregunta. 


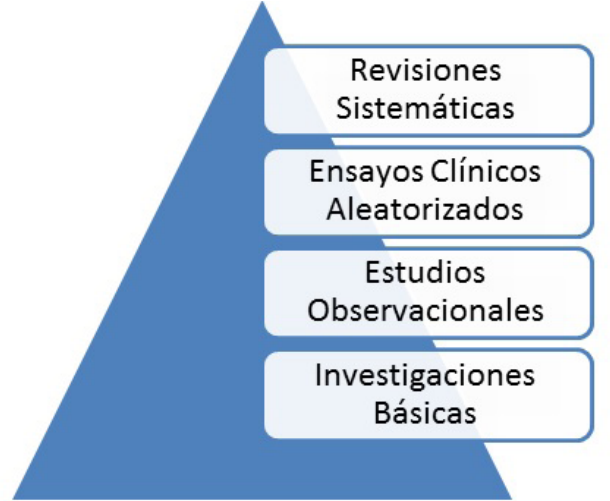

Figura 1. Jerarquía de la evidencia.

La búsqueda, debe ser realizada por dos autores de manera paralela en las bases de datos disponibles, en literatura gris y expertos.

Una vez obtenida la información, se deben seleccionar los artículos y, a partir de los seleccionados, se obtendrán los datos y se realizarán los análisis críticos y estadísticos de la información, finalmente exponiendo los resultados del trabajo (Figura 2).

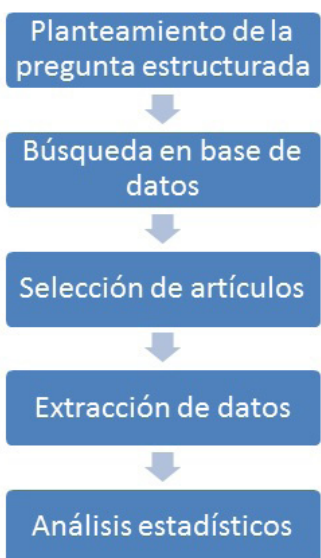

Figura 2. Proceso de elaboración de una revisión sistemática.

1. Planteamiento de la pregunta estructurada. Toda búsqueda de información parte con la aparición de una incógnita para el clínico tratante, a partir de la cual se determinará una pregunta clínica. Dicha pregunta debe ser clara y precisa, orientada a outcomes clínicamente significativos para el paciente y debe estar conformada por cinco elementos esenciales:

- Población: definición de un grupo determinado de la población según factores como: edad, sexo, raza o la presencia de una condición especial de interés.

- Intervención: procedimiento que se desea estudiar.

- Comparación: intervención o ausencia de la misma con la que se comparará.

- Outcome: evento clínico o desenlace de la intervención en estudio.

- Tipo de estudio: en el caso de las preguntas de terapia, corresponden a ensayos clínicos aleatorizados.

Lo anteriormente mencionado corresponde al enfoque PICO y permite estructurar de manera correcta la pregunta y definir los conceptos con los que se realizará la búsqueda ${ }^{(5)}$.

2. Búsqueda en base de datos. En primer lugar, se deben definir los criterios de elegibilidad de acuerdo al enfoque PICO y el tipo de estudios que serán incluidos. A continuación, se debe explicitar la estrategia de búsqueda que se ejecutará para realizar la detección de artículos atingentes a la pregunta PICO, así como dónde se llevará a cabo esta búsqueda, estableciendo a priori, la metodología de investigación y facilitando la reproducibilidad del trabajo. Es recomendable utilizar términos de múltiples formas para referirse a la misma situación, a fin de captar el mayor número de trabajos potencialmente elegibles, bases de datos como MEDLINE o EMBASE ofrecen herramientas como los términos MeSH o EMTREE, los cuales reúnen diferentes términos que se refieren al mismo concepto en la literatura. Con los términos anteriormente definidos se debe realizar la búsqueda de artículos atingentes a la pregunta clínica. La búsqueda, debe ser realizada a lo menos por dos participantes independientes, de manera que sea un proceso lo más objetivo posible y se disminuya al máximo la posibilidad de sesgos. Los resultados obtenidos de la búsqueda deben ser expuestos en forma clara. La búsqueda debe ser realizada en bases de datos electrónicas, de forma manual en revistas, en literatura gris e incluso con expertos en el área, de manera que no se omita información.

3. Selección de los artículos. La selección inicial se realiza en base a los resúmenes y títulos de la información disponible identificando los artículos potencialmente elegibles. Si durante el proceso existe alguna discrepancia entre los participantes, se debe llegar a un consenso u otro participante debe ayudar a dirimir. Una vez definidos, se analizan en su totalidad y por completo los artículos seleccionados y se realiza una selección final a través de criterios de inclusión que permiten analizar críticamente los artículos y así obtener los que respondan claramente la pregunta clínica planteada anteriormente. Resulta útil el uso de diagramas de flujo para presentar el proceso de selección de artículos, donde se especifica desde el número inicial de artículos potencialmente elegibles según la búsqueda realizada hasta los finalmente incluídos, especificando los motivos por los cuales se excluyeron los trabajos que no se consideraron finalmente (Figura 3 ).

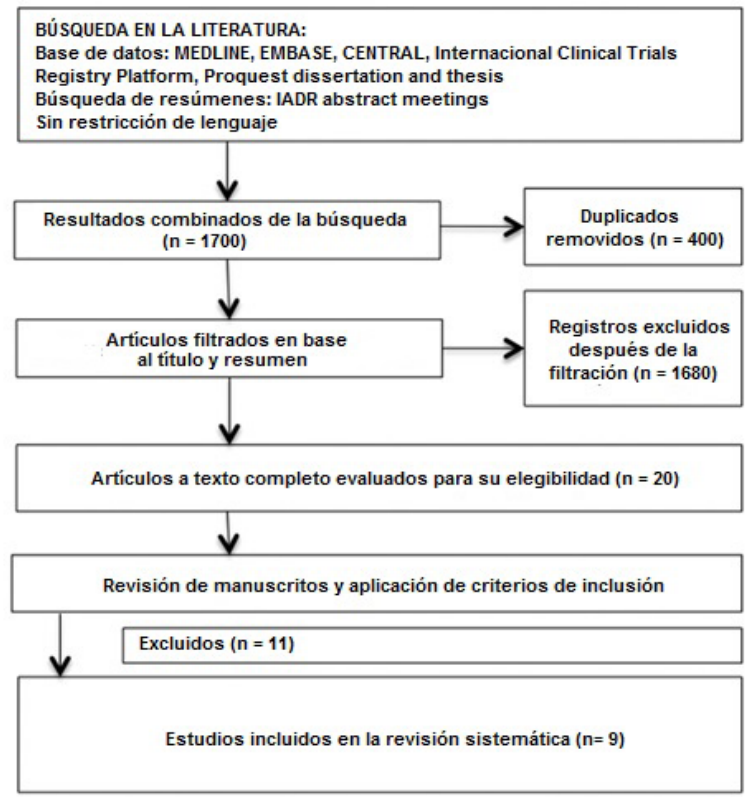

Figura 3. Ejemplo de diagrama de flujo de selección de artículos.

4. Extracción de datos. Una vez finalizada la selección de los artículos, se debe obtener de ellos toda la información atingente a la pregunta: cómo se realizó el estudio, quiénes y cuántos participaron, cuál fue la intervención, cuáles fueron los resultados medidos, cuáles fueron las fuentes de financiamiento, etc. Los datos deben ser tabulados en un formulario de recolección de datos. Además de los datos mencionados, se debe evaluar el riesgo de sesgo de los artículos, ya que las conclusiones de la revisión sistemática podrán ser válidas en la medida en que los estudios que la componen, llamados estudios primarios, sean confiables (Figura 4).

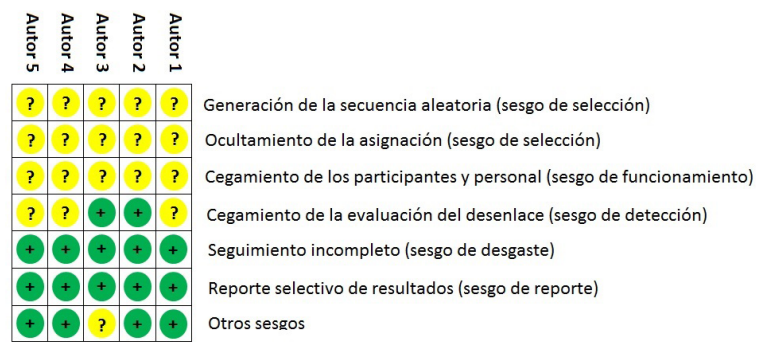

Figura 4. Ejemplo de evaluación del riesgo de sesgo de los artículos incluidos en una revisión. 
5. Análisis estadístico. Los resultados obtenidos en cada artículo estudiado pueden compararse y resumirse a través de análisis estadísticos. En las revisiones sistemáticas el método más utilizado son los metanálisis, que corresponden a un análisis estadístico de los resultados de estudios independientes que generalmente intenta producir un estimador único del efecto de la intervención estudiada. La decisión de realizar o no un meta-análisis en la revisión sistemática debe ser evaluada en cada caso y debe considerar el tipo y calidad de los datos obtenidos previamente en la revisión misma, ya que no siempre es favorable su realización. Debe considerarse que el fin último de la revisión sistemática es proveer una herramienta práctica para la toma de decisiones clínicas, por lo que la presentación de los resultados es crucial para su utilidad. Los resultados del metanálisis son graficados en un forest plot (o diagrama de bosque) en el cual se pueden observar típicamente (Figura 5):

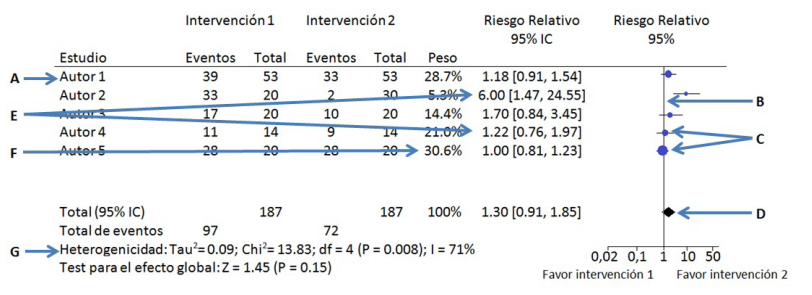

Figura 5. Ejemplo de meta-análisis de una revisión sistemática.

- Identificador del estudio: ya sea autor, nombre del estudio, año de publicación, etc. (A)

- Línea vertical de no efecto: es decir, el punto donde no existen diferencias entre los grupos que se están comparando. (B)

- Estimador puntual y su intervalo de confianza al 95\% de cada estudio (C)

- Estimador puntual global y su intervalo de confianza al 95\%, que corresponde al rombo observado al final del diagrama (D)

- Medidas de asociación utilizada y estimaciones puntuales exactas e intervalos de confianza de cada estudio en cifras (E)

- Peso de cada artículo incluido en el estudio, expresado en porcentaje (F)

- Test estadísticos y estadístico I2 para evaluar la heterogeneidad (G)

Una vez obtenidos los elementos del análisis estadístico de los artículos incluidos en la revisión, cada uno permite obtener conclusiones respecto a la información recopilada:

- el estimador global permite observar el resultado final de los estudios incorporados, favoreciendo una u otra intervención según el lado de la línea de no efecto en que se encuentre;

- su intervalo de confianza permite establecer que tan exacto es el resultado y, por lo tanto, establece cierto grado de seguridad de dicho resultado;

- el peso de cada uno de los estudios entrega la información sobre qué articulo incorporado tiene mayor valor según su tamaño

- el test estadístico I2, o el test de heterogeneidad utilizado, permiten observar que tan variables son los resultados de los estudios incorporados entre sí.

De esta manera, podemos observar que cada elemento obtenido en el análisis estadístico de la revisión tiene un rol crucial y es necesario para establecer la aplicabilidad de ésta a la práctica clínica.

\section{CONCLUSIÓN.}

Las revisiones sistemáticas facilitan y resumen los contenidos de múltiples artículos que responden una misma pregunta clínica. Dado su estricto protocolo de elaboración corresponden al mejor nivel de evidencia ya que disminuyen al máximo el riesgo de sesgo, permitiendo, de esta forma, tomar decisiones clínicas informadas basadas en evidencia.

\section{CONFLICTOS DE INTERÉS}

Los autores declaran no tener conflictos de interés, ni fuentes de financiamiento.

\section{Bibliografía}

1. Brignardello-Petersen R, Carrasco-Labra A, Glick M, Guyatt GH, Azarpazhooh A A practical approach to evidence-based dentistry: understanding and applying the principles of EBD. J Am Dent Assoc. 2014;145(11)1105-1107.

2. Rada G, Letelier L. ¿Podemos mantenernos actualizados en medicina en el siglo XXI? Rev Méd Chile 2009; 137(5)701-708.

3. Letelier L, Manriquez J, Rada G. Revisiones sistemáticas y metaanálisis: ¿son la mejor evidencia?. Rev Méd Chile 2005; 133(2)246-249.

4. Carrasco-Labra A, Brignardello-Petersen R, Glick M, Guyatt GH, Azarpazhooh A. A practical approach to evidence-based dentistry: VI. How to use a systematic review. J Am Dent Assoc. 2015; 146(4)255-265.
5. Guyatt G, Meade MO, Richardson S, Jaeschke R. What is the question? In: Guyatt G, Rennie D, Meade MO, Cook DJ, eds. Users' Guides to the Medical Literature: A Manual for Evidence-Based Clinical Practice. 2nd ed. New York City: McGraw-Hill; 2008:17-28

6 Higgins JPT, Green S (editors). Cochrane Handbook for Systematic Reviews of Interventions Version 5.1.0 [updated March 2011]. The Cochrane Collaboration, 2011. Available from www.cochrane-handbook.org. 